\title{
The changing trends in antibiotic resistance pattern in septicemia.
}

\author{
Pavani Nimmala $^{1}$, Parveen Anjum ${ }^{2}$, V.Aruna Rajkumari ${ }^{3}$ \\ Department of Microbiology, Chelmeda Anandrao Institute of Medical Sciences, Karimnagar, Andhra Pradesh, \\ India.
}

\begin{abstract}
Septicemia is one of the important causes of morbidity and mortality. Emergence of multidrug resistant bacteria is a major problem in the management of septicemia. Present study was undertaken to isolate the common bacterial pathogens associated with septicemia and determine their antibiotic resistance. Blood cultures from 100 suspected cases of septicemia were examined. The growths from the subcultures were identified by conventional biochemical tests. Antibiotic susceptibility testing done by Kirby-Bauer disk diffusion method and antibiotic resistant strains in the primary screening were further processed for MRSA, ESBL and MBL production. Out of 100 suspected cases, 28 (28\%) were cultures positive. Of which 15 (53.57\%) were Gram positive cocci and 13 (46.42\%) were Gram negative bacilli. The prevalence of MR CONS was found to be high $78 \%$ when compared to MRSA 67\%. The overall prevalence of ESBL producers among Gram negative bacilli was found to be $46 \%$ and MBL among Pseudomonas aeruginosa was 33\%.Thus, the study highlights the rising level of antibiotic resistance amongst the bacterial isolates from blood and, hence, the need to continuously monitor the locally emerging antibiotic resistance patterns, and updates the existing antibiotic policies.
\end{abstract}

Key Words: Septicemia, antibiotic resistance.

\section{Introduction}

Antibiotic resistance is an issue of great significance for public health at the global level. The spread of drug resistant pathogens is one of the most serious threats to the successful treatment of microbial disease ${ }^{1}$. Nowadays, about $70 \%$ of bacteria that cause infections in hospitals are resistant to atleast one of the antibiotic agents most commonly used for treatment ${ }^{2}$.

Septicemia is a major cause of morbidity and mortality in the world and associated with longer hospitalization and elevated $\operatorname{cost}^{3}$. Owing to a high mortality in septicemia, prompt initiation of appropriate antimicrobial therapy is demonstrably important. Initial therapy must be empirically based on the likely pathogens and typical patterns of antimicrobial susceptibility ${ }^{4}$.

Over the years, undisciplined use of broad spectrum antibiotics, prolonged courses of antibiotic therapy have resulted in increased incidence of extended-spectrum beta-lactamase (ESBL), methicillin-resistant Staphylococcus aureus (MRSA), Vancomycin-resistant Enterococci, Carbepenem-resistant Pseudomonas/ Acinetobacter and multi-drug-resistant bacteria ${ }^{5}$.

Since early 1950's, there is a striking increase in the incidence of bacteraemia caused by members of Enterobacteriaceae and other Gram negative bacilli. Escherichia coli which was reported to be the most common in past is being replaced by other multidrug resistance bacteria like Klebsiella, Enterobacter, Salmonella, Citrobacter, Pseudomonas, Acinetobacter ${ }^{6}$. In the 1980's and 1990's, the pattern shifted so that most bacteraemia were due to Gram positive organisms such as Staphylococcus aureus, CONS, Enterococcus $\mathrm{spp}^{3}$. In addition to the shift in organisms responsible for bacteraemia, the susceptibility patterns of pathogens causing bacteraemia have also changed ${ }^{7}$.

In the present study we determine the prevalence of antibiotic resistance among the isolates causing septicemia.

\section{Material and Methods}

The present study was carried out between June 2010 to May 2011 in the Department of Microbiology, CAIMS, Karimnagar. A total number of 100 patients of all age groups who presented with clinical impression of septicemia or prolonged fever were included in the study.

Blood samples were collected for culture under strict aseptic precautions and inoculated into brain heart infusion broth. Subcultures on to blood agar and macconkey agar plates were then made after $24 \mathrm{hrs}, 48 \mathrm{hrs}$ and 72hrs. Organisms were identified by standard methods of identification. ${ }^{8}$

Antibiotic susceptibility testing was done by Kirby Bauer disc diffusion method. Zone size measured and interpreted according to CLSI standards. ${ }^{9}$ Antibiotic resistant strains in primary screening was further processed for detection of methicillin resistance Staphylococcus aureus (MRSA), extended spectrum beta 
The changing trends in antibiotic resistance pattern in septicemia.

lactamases (ESBL) in Gram negative bacterial isolates and metallo beta lactamases (MBL) in Pseudomonas aeruginosa.

Detection of MRSA was done by Oxacillin disc $(1 \mu \mathrm{g})$ on the bacterial lawn culture of S aureus. After overnight incubation, the zone of inhibition was measured. An inhibition zone diameter less than or equal to $10 \mathrm{~mm}$ indicates MRSA. ${ }^{10}$

ESBL producers detected by combination disk method using Ceftazidime $(30 \mu \mathrm{g})$ and ceftazidime + clavulunic acid $(20 \mu \mathrm{g}+10 \mu \mathrm{g})$ were placed at the distance of $20 \mathrm{~mm}$ from center to center. Plates were incubated at $37^{\circ} \mathrm{C}$ overnight. A $>5 \mathrm{~mm}$ increase in zone diameter for the antimicrobial tested in combination with clavulanic acid versus its zone when tested alone confirmed ESBL production. ${ }^{11}$

Imipenem resistant Pseudomonas aeruginosa isolates were further screened for MBL production .Imipenem-Ethylene diamine tetra acetic acid (EDTA) double disc synergy test:Test organisms were inoculated on the plates with Muller Hinton agar .An imipenem (10 microgram) disc was placed 20mm centre to centre from blank disc containing 10 microL of 0.5M EDTA (750 microgram). Enhancement of the zone of inhibition in the area between imipenem and the EDTA discs in comparison with the zone of inhibition on the far side of the drug was interpreted as positive. ${ }^{12}$

\section{Results}

Out of 100 clinically suspected cases of septicaemia $28(28 \%)$ were blood culture positive. the incidence of septicaemia was high in neonates (45\%) followed by infants (33\%) and >60yrs (33\%).

The incidence of Gram positive organism is more (53.57\%) when compared to the Gram negative organisms $(46.42 \%)$. The most common organism isolated were Coagulase Negative Staphylococci 9 (32.10\%) followed Staphylococcus aureus $6(21.42 \%)$, Klebsiellapneumoniae 5 (17.85\%), Pseudomonas aeruginosa and Citrobacterfreundii 3 each (10.71\%) and Escherichia coli and Proteus mirabilis 1 each (3.57\%) .

Table 1 shows Antibiotic resistance pattern in Gram positive cocci with CONS showing multidrug resistance when compared to Staphylococcus aureus.

\begin{tabular}{|l|c|c|}
\hline ANTIBIOTICS & CONS & S.AUREUS \\
\hline AMPICILLIN & $89 \%$ & $83 \%$ \\
\hline AMOXYCLAV & $44 \%$ & $33 \%$ \\
\hline ERYTHROMYCIN & $68 \%$ & $65 \%$ \\
\hline VANCOMYCIN & $0 \%$ & $0 \%$ \\
\hline LINEZOLID & $0 \%$ & $0 \%$ \\
\hline PIPERACILLIN/TAZOBACTAM & $22 \%$ & $18 \%$ \\
\hline AMIKACIN & $44 \%$ & $44 \%$ \\
\hline GENTAMYCIN & $54 \%$ & $50 \%$ \\
\hline CEPHOTAXIME & $67 \%$ & $62 \%$ \\
\hline CIPROFLOXACIN & $56 \%$ & $40 \%$ \\
\hline
\end{tabular}

Table 1: Antibiotic Resistance in Gram positive cocci

Table 2 shows Antibiotic resistance pattern in Gram negative bacilli.

\begin{tabular}{|l|c|c|c|c|c|}
\hline ANTIBIOTICS & K.PNEUMONIAE & C.FREUNDII & E.COLI & P.MIRBILIS & P.AERUGINOSA \\
\hline AMPICILLIN & $84 \%$ & $100 \%$ & $100 \%$ & $100 \%$ & NT \\
\hline PIPERACILLIN/ & $38 \%$ & $33 \%$ & $0 \%$ & $0 \%$ & $33 \%$ \\
TAZOBACTAM & & & & & $67 \%$ \\
\hline AMIKACIN & $56 \%$ & $33 \%$ & $0 \%$ & $0 \%$ & $67 \%$ \\
\hline GENTAMYCIN & $58 \%$ & $67 \%$ & $100 \%$ & $100 \%$ & $67 \%$ \\
\hline CEPHOTAXIDIME & $78 \%$ & $67 \%$ & $100 \%$ & $100 \%$ & $67 \%$ \\
\hline CEFTRIAXONE & $54 \%$ & $33 \%$ & $100 \%$ & $0 \%$ & $67 \%$ \\
\hline CEFIPIME & $76 \%$ & $33 \%$ & $0 \%$ & $0 \%$ & $0 \%$ \\
\hline CIPROFLOXACIN & $40 \%$ & $33 \%$ & $0 \%$ & $0 \%$ & $33 \%$ \\
\hline IMIPENEM & $60 \%$ & $67 \%$ & $100 \%$ & $100 \%$ & $67 \%$ \\
\hline
\end{tabular}

(NT-Not Tested)

Table 2: Antibiotic Resistance pattern in Gram negative bacilli 
The changing trends in antibiotic resistance pattern in septicemia.

Table 3 shows the incidence of ESBLs

\begin{tabular}{|l|r|r|}
\hline ORGANISMS & \multicolumn{1}{|l|}{ TOTAL ISOLATES } & \multicolumn{2}{|l|}{ ESBL ISOLATES } \\
\hline K.PNEUMONIAE & 5 & 3 \\
\hline C.FREUNDII & 3 & 1 \\
\hline ESCH. COLI & 1 & 1 \\
\hline P.MIRABILIS & 1 & - \\
\hline P.AERUGINOSA & 3 & 1 \\
\hline TOTAL & 13 & 6 \\
\hline
\end{tabular}

Table 3 :The incidence of ESBLs

The antibiotic resistance pattern in ESBL producers which showed resistance to three or more drugs than the non- ESBL producers. (100\%) ESBL producers were resistant to Ampicillin, (83.3\%) resistance against Gentamycin and Ciprofloxacin, (66.6\%) against Amikacin and Imipenem.

\section{Discussion}

The bacteriological profile with antibiotic susceptibility pattern plays an important role in the effective management of septicemia. Out of 100 blood samples collected from clinically suspected cases of septicemia, $28(28 \%)$ were blood culture positive which is comparable to the previous studies. ${ }^{13}$

In the present study the incidence of septicemia was more in neonates $45 \%$ and Infants and over 60 years $33 \%$. This is due to the under developed immunity in neonates and infants and decreased immunity in age above 60 years.

In 28 isolates from blood cultures Gram positive organisms were the main pathogens $15(53.57 \%)$ which is in accordance with previous studies. ${ }^{14}$ Coagulase negative Staphylococci 9 (32.14\%) and Staphylococcus aureus $6(21.42 \%)$ being the most frequent Gram positive organisms isolated in the present study.

Though not all positive blood cultures of CONS represent true bacteremia because CONS in the skin flora commonly causes contamination of blood cultures. This makes the evaluation of a positive blood culture difficult in individual patients. A true bacteremia was defined as either the growth of the same strain of CONS in sequential blood cultures or a positive blood culture accompanied by clinical signs of sepsis. ${ }^{15}$

CONS are now being recognized as the most common cause of nosocomial infections in intensive care units. More in the incidence of Coagulase negative Staphylococci could be due to increasing use of intravascular devices in the medical care.

In the present study CONS occupy the first place in infection of neonatal blood. The isolated ratio of MRCONS is high and shows multi-drug resistance when compared to MRSA. The high percentages of methicillin resistance results in frequent use of glycopeptides, third-generation cephalosporins and fluoroquinolones. Among CONS and Staphylococcus aureus resistance rates were highest against ampicillin followed by erythromycin, cephotaxime, amikacin and ciprofloxacin. In the present study there was no resistance towards Vancomycin and linezolid .

In Gram negative organisms Klebsiellapneumoinae, Citrobacterfreundii, Escherichia coli showed high resistance to ampicillin, third generation cephalosporins, amikacin and ciprofloxacin. Pseudomonas aeruginosa was resistant to third generation cephalosporins and amikacin.

The alarming finding in the present study was the resistance in Gram negative bacilli to $3^{\text {rd }}$ generation Cephalosporins ranging from 54\% to $64 \%$. This type of resistance was a marker for the presence of ESBL.

ESBL production in the present study was (46\%). Klebsiella pneumoniae were the largest ESBL producers accounting (60\%) followed by Citrobacterfreundii and Pseudomonas aeruginosa (33\%). Only one Escherichia coli was isolated which is also an ESBL producer.

Carbapenems are considered as the last resort for ESBL producing isolates and resistance to Carbapenems is a serious concern. In this study, one isolate of Klebsiella pneumoniae and one isolate of Pseudomonas aeruginosa producing ESBL were resistant to Imipenem. The resistance may be due to reduce levels of drug accumulation or increased expression of pump efflux or may be due to the production of metallo betalacatamase in Pseudomonas spp.

The pathogens isolated in this study showed highly multiple drug resistances. There was resistance against vancomycin and linezolid for Gram positive organisms and low rates of resistance against imipenem and cefipime for Gram negative organisms. Piperacillin/tazobactam susceptibility was high for both Grampositive and Gram-negative organisms 


\section{Conclusion}

The changing spectrum of microbial pathogens and rise in antibiotic resistance in septicemia emphasise the importance of hospital infection control, regular antibiotic susceptibility surveillance and evaluation, and the enforcement and periodic review of the antibiotic policy of the hospital as well as the encouragement of rational antibiotic use, a key point in controlling the occurrence and spread of resistance. In view of the isolation of highly resistant organisms in this study, imipenem and piperacillin/tazobactam is suggested to be the drug of choice for empirically treating Gram negative septicemia and linezolid, vancomycin for Gram positive Septicemia. Prompt and effective therapy requires up to date knowledge of locally prevalent organisms, and an on-going surveillance for emerging antibiotic resistance. Routine screening for MRSA, ESBL and MBL needs to be included in all the clinical laboratories to ensure a favourable clinical outcome. 